\title{
Aortic dissection in patients with Marfan syndrome based on the IRAD data
}

\author{
Hector W. L. de Beaufort ${ }^{1}$, Santi Trimarchi ${ }^{1}$, Amit Korach ${ }^{2}$, Marco Di Eusanio $^{3}$, Dan Gilon ${ }^{4}$, Daniel G. \\ Montgomery ${ }^{5}$, Arturo Evangelista ${ }^{6}$, Alan C. Braverman ${ }^{7}$, Edward P. Chen ${ }^{8}$, Eric M. Isselbacher ${ }^{9}$, Thomas \\ G. Gleason ${ }^{10}$, Carlo De Vincentiis ${ }^{1}$, Thoralf M. Sundt ${ }^{11}$, Himanshu J. Patel ${ }^{12}$, Kim A. Eagle
}

${ }^{1}$ Thoracic Aortic Research Center, Policlinico San Donato IRCCS, University of Milan, San Donato Milanese, Italy; ${ }^{2}$ Department of Cardiothoracic Surgery, Hadassah Hebrew University Medical Center, Jerusalem, Israel; ${ }^{3}$ Department of Cardiac Surgery, Ospedali Riuniti di Ancona, Ancona, Italy; ${ }^{4}$ Department of Cardiology, Hadassah Hebrew University Medical Center, Jerusalem, Israel; ${ }^{5}$ Department of Medicine, Frankel Cardiovascular Center, University of Michigan, Ann Arbor, MI, USA; ${ }^{6}$ Department of Cardiology, Hospital General Universitari Vall d'Hebron, Barcelona, Spain; ${ }^{7}$ Cardiovascular Division, Department of Medicine, Washington University of Medicine, Saint Louis, MO, USA; ${ }^{8}$ Division of Cardiothoracic Surgery, Emory University, Atlanta, GA, USA; ${ }^{9}$ Thoracic Aortic Center and Cardiology Division, Massachusetts General Hospital and Harvard Medical School, Boston, MA, USA; ${ }^{10}$ Division of Cardiac Surgery, Department of Cardiothoracic Surgery, University of Pittsburgh School of Medicine, Pittsburgh, PA, USA; ${ }^{11}$ Division of Cardiac Surgery, Department of Surgery, Massachusetts General Hospital and Harvard Medical School, Boston, MA, USA; ${ }^{12}$ Department of Cardiac Surgery, Frankel Cardiovascular Center, University of Michigan, Ann Arbor, MI, USA Correspondence to: Santi Trimarchi, MD, PhD. Thoracic Aortic Research Center, Policlinico San IRCCS, University of Milan, Piazza Malan 2, 20097 San Donato Milanese, Italy. Email: santi.trimarchi@grupposandonato.it.

Between January 1996 and May 2017, the International Registry on Acute Aortic Dissections has collected information on a total of 6,424 consecutive patients with acute aortic dissection, including 258 individuals with a diagnosis of Marfan syndrome. Patients with Marfan syndrome presented at a significantly younger age compared to patients without Marfan syndrome $(38.2 \pm 13.2$ vs. $63.0 \pm 14.0$ years; $\mathrm{P}<0.001)$ and in general had fewer comorbidities, although they more frequently had a known aortic aneurysm and history of prior cardiac surgery. We noted significantly larger diameters of the aortic annulus and root in the Marfan syndrome cohort, but no larger diameters more distally. The in-hospital mortality in type A dissection was not significantly different in patients with or without Marfan syndrome, despite the differences in age and comorbidities and the lower incidence of aortic rupture in the Marfan syndrome cohort. In contrast, the in-hospital mortality of Marfan syndrome patients with type B dissection appears to be lower than that of patients without Marfan syndrome. The Marfan syndrome cohort that was treated with open surgery for type B dissection seemed to do especially well, with a $0 \%$ mortality rate $(\mathrm{n}=27)$. Follow-up data for type A and B dissections combined show an estimated five-year survival rate of $80.1 \%$ and an estimated reintervention rate of $55.3 \%$ in patients with Marfan syndrome. Such a high rate of reinterventions highlights the need for careful surveillance and treatment for patients with Marfan syndrome surviving the acute phase of aortic dissection.

Keywords: Aortic dissection; Marfan syndrome; aortic surgery; operative results

Submitted Jun 21, 2017. Accepted for publication Oct 09, 2017.

doi: 10.21037 /acs.2017.10.03

View this article at: http://dx.doi.org/10.21037/acs.2017.10.03 


\section{Introduction}

Marfan syndrome is a heritable disorder of the fibrillin 1 (FBN1) gene, which encodes the connective tissue protein fibrillin-1. In most patients, the connective tissue disorder leads to abnormalities of the aortic wall, causing progressive aortic dilatation, thus increasing the risk of acute aortic dissection. Aortic root dilatation/dissection is in fact one of the cardinal features of Marfan syndrome, according to the revised Ghent criteria (1). Surgical replacement of the dilated aortic root and ascending aorta has significantly increased the life expectancy of patients with Marfan syndrome $(2,3)$. Nevertheless, aortic dissection remains the leading cause of morbidity and mortality in these patients (2).

The International Registry of Acute Aortic Dissection (IRAD) represents an investigational collaboration that has collected information on unselected consecutive cases of acute aortic dissection occurring at 30 aortic referral centers in 10 countries since January 1, 1996. The rationale and structure of IRAD have been published previously (4). A number of observations on Marfan syndrome have been made in previous IRAD studies (5-8). To gain additional insight into surgical strategies and outcomes for the cohort of Marfan syndrome patients enrolled in IRAD, an updated analysis of the database was performed.

\section{Methods}

For the current analysis, the characteristics of the cohort of Marfan patients were compared to those of all other enrolled patients in IRAD. In IRAD, the diagnosis of Marfan syndrome was made at each study site, but was not independently verified by a central mechanism. Continuous variables are expressed as mean \pm standard deviation $(\mathrm{SD})$ or median and interquartile range (IQR). Categorical variables are expressed as percentages. In all cases, missing data were not defaulted to negative and denominators reflect only cases reported. Univariate analyses between groups were done using Chi-square tests (or Fisher exact tests) and Student's $t$-tests where appropriate. For KaplanMeier survival estimates, groups were compared with a $\log$ rank test. All $\mathrm{P}$ values are two-sided, with values $<0.05$ considered as significant. Statistical analysis was performed using SPSS 22.0.

\section{Demographics, comorbidities and presentation}

A total of 6,424 consecutive patients with acute aortic dissection were enrolled in IRAD between January 1996 and May 2017, including 258 individuals (4\%) with a diagnosis of Marfan syndrome. Patients with Marfan syndrome presented at a significantly younger age than patients without Marfan syndrome $(38.2 \pm 13.2$ vs. $63.0 \pm 14.0$ years; $\mathrm{P}<0.001)$. The difference in age applied for type A dissections $(36.9 \pm 13.2$ vs. $62.2 \pm 14.1, \mathrm{P}<0.001)$ as well as for type B dissections $(40.3 \pm 12.9$ vs. $64.3 \pm 13.7, \mathrm{P}<0.001)$. Patients with Marfan syndrome had a lower incidence of hypertension, atherosclerosis and diabetes mellitus, while they more frequently had a known aortic aneurysm and had more frequently undergone prior cardiac surgery (Table 1). The incidence of bicuspid valve was not significantly different between both groups $(3.8 \%$ vs. $3.0 \%$; $\mathrm{P}=0.527)$. Patients with Marfan syndrome presented with a Stanford type A dissection in $63.6 \%$ of cases and a type $\mathrm{B}$ dissection in $36.4 \%$ of cases. The distribution of type A and $\mathrm{B}$ dissection was not significantly different between patients with or without Marfan syndrome $(\mathrm{P}=0.691)$. The incidence of pregnancy-associated aortic dissection in Marfan syndrome patients $(62.5 \%$ of which were type A and $27.5 \%$ type B dissections) was significantly higher compared to patients without Marfan syndrome $(3.3 \%$ vs. $0.3 \% ; \mathrm{P}<0.001)$.

The median diameters of the aortic annulus and root were significantly larger in patients with Marfan syndrome compared to patients without Marfan syndrome, while there were no significant differences in the diameters of the sinotubular junction, ascending aorta, or descending aorta (Table 2). Marfan patients more frequently had aortic regurgitation than those without Marfan syndrome (54.5\% vs. $37.1 \% ; \mathrm{P}<0.001)$, more frequently had coronary artery involvement of the dissection $(13.1 \%$ vs. $8.2 \% ; \mathrm{P}=0.033)$ and less frequently presented with pericardial effusion $(19.6 \% \mathrm{vs}$. $29.8 \% ; \mathrm{P}=0.002)$ or periaortic hematoma ( $9.8 \%$ vs. $16.5 \%$; $\mathrm{P}=0.018$ ). The false lumen was more frequently fully patent in patients with Marfan syndrome than in those without $(76.4 \%$ vs. $62.3 \% ; \mathrm{P}<0.001)$ and correspondingly, less frequently completely thrombosed $(2.5 \%$ vs. $11.3 \% ; \mathrm{P}<0.001)$. There was no significant difference in the frequency of partial false lumen thrombosis ( $21.1 \%$ vs. $26.4 \%$; $\mathrm{P}=0.138)$.

The clinical presentation of patients with Marfan syndrome is shown in Table 3. This shows that patients with Marfan syndrome presented less frequently with complications of stroke $(1.3 \%$ vs. $3.8 \%, \mathrm{P}=0.051)$, acute renal failure $(5.5 \%$ vs. $11.0 \%, \mathrm{P}=0.007)$, and limb ischemia (6.5\% vs. $12.5 \%, \mathrm{P}=0.005$ ), but more frequently with aortic insufficiency grade 3 or $4(14.6 \%$ vs. $7.0 \%, \mathrm{P}<0.001)$. 


\begin{tabular}{|c|c|c|c|}
\hline Characteristics & Marfan syndrome $(n=258)$ & No Marfan syndrome $(n=6,166)$ & $P$ value \\
\hline Age $($ mean $\pm S D)$ & $38.2 \pm 13.2$ & $63.0 \pm 14.0$ & $<0.001$ \\
\hline Gender, female & $92(35.7 \%)$ & $2,115(34.3 \%)$ & 0.653 \\
\hline Race, non-white & $49(20.2 \%)$ & $1,078(18.7 \%)$ & 0.535 \\
\hline \multicolumn{4}{|l|}{ Type of dissection } \\
\hline Type A dissection & $164(63.6 \%)$ & $3,994(64.8 \%)$ & 0.691 \\
\hline Type B dissection & $94(36.4 \%)$ & $2,172(35.2 \%)$ & 0.691 \\
\hline Hypertension & $103(41.9 \%)$ & $4,809(78.4 \%)$ & $<0.001$ \\
\hline Atherosclerosis & $16(6.8 \%)$ & $1,393(23.0 \%)$ & $<0.001$ \\
\hline Known aortic aneurysm & $89(37.6 \%)$ & 909 (14.9\%) & $<0.001$ \\
\hline Diabetes mellitus & $10(4.3 \%)$ & $541(8.9 \%)$ & 0.014 \\
\hline Bicuspid aortic valve & $8(3.8 \%)$ & $169(3.0 \%)$ & 0.527 \\
\hline Any prior cardiac surgery & $107(44.8 \%)$ & $835(14.2 \%)$ & $<0.001$ \\
\hline Aortic valve replacement & $68(28.8 \%)$ & $218(3.7 \%)$ & $<0.001$ \\
\hline Mitral valve replacement & $9(3.8 \%)$ & $50(0.9 \%)$ & $<0.001$ \\
\hline Aortic aneurysm surgery & $88(37.4 \%)$ & $463(7.9 \%)$ & $<0.001$ \\
\hline CABG & $10(4.3 \%)$ & $286(4.9 \%)$ & 0.705 \\
\hline Pregnancy-associated aortic dissection & $8(3.3 \%)$ & $15(0.3 \%)$ & $<0.001$ \\
\hline
\end{tabular}

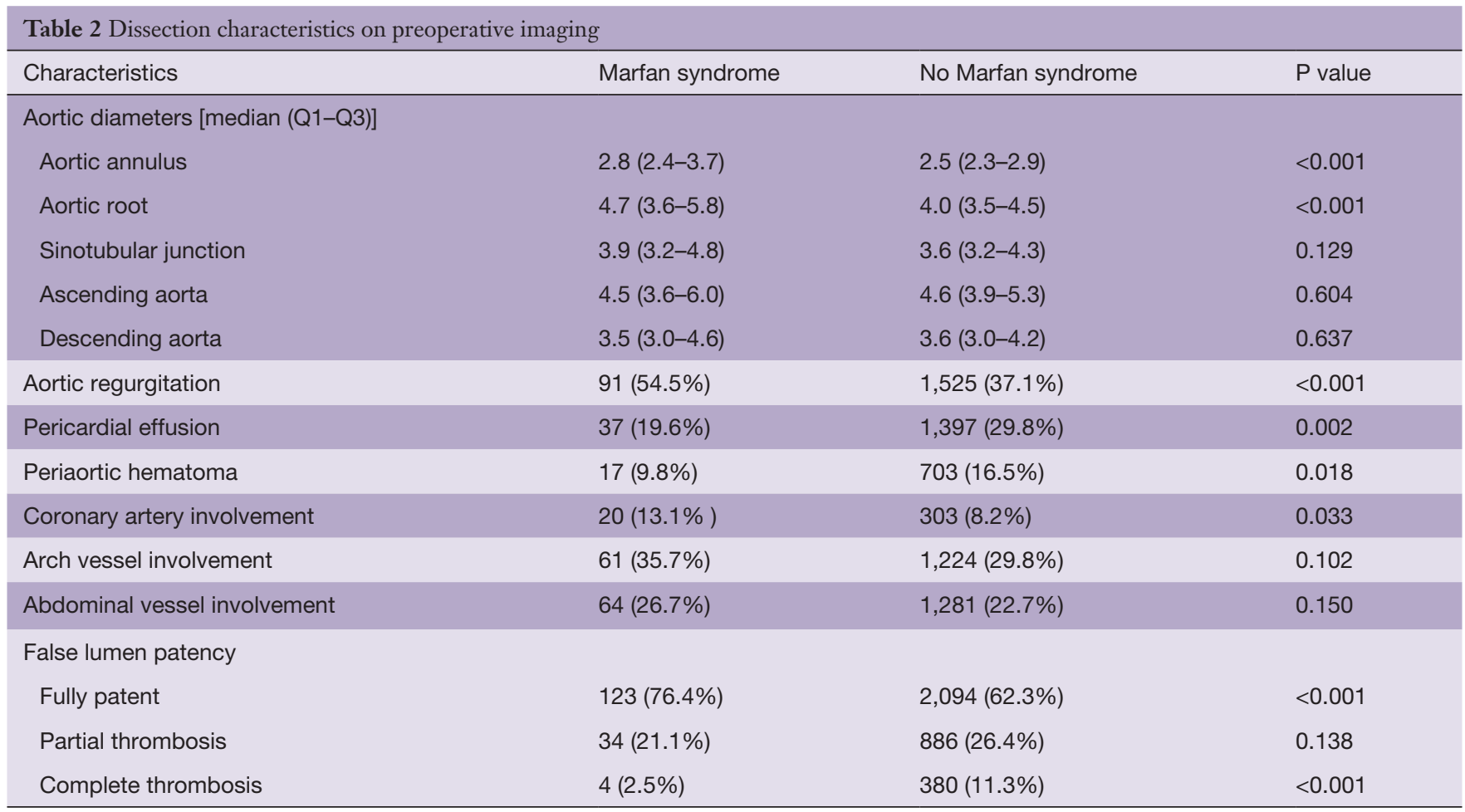




\begin{tabular}{|c|c|c|c|}
\hline Hours from onset of symptoms to diagnosis, median (IQR) & $4.1(2.3-9.1)$ & $5.0(3.0-12.0)$ & 0.014 \\
\hline $\begin{array}{l}\text { Hours from onset of symptoms to intervention for type A dissections, } \\
\text { median (IQR) }\end{array}$ & $8.2(5.5-28.1)$ & $9.5(6.0-20.5)$ & 0.117 \\
\hline Shock & $19(8.3 \%)$ & $352(6.5 \%)$ & 0.293 \\
\hline Congestive heart failure & $19(8.2 \%)$ & $306(5.4 \%)$ & 0.074 \\
\hline Aortic insufficiency grade 3 or 4 & $30(14.6 \%)$ & $336(7.0 \%)$ & $<0.001$ \\
\hline Cardiac tamponade & $15(6.3 \%)$ & $467(8.2 \%)$ & 0.309 \\
\hline Visceral ischemia & $12(5.1 \%)$ & $294(5.1 \%)$ & 0.968 \\
\hline Acute renal failure & $13(5.5 \%)$ & $629(11.0 \%)$ & 0.007 \\
\hline Limb ischemia & $16(6.5 \%)$ & $753(12.5 \%)$ & 0.005 \\
\hline
\end{tabular}

*, myocardial complications include: acute myocardial infarction, myocardial ischemia, preoperative low output syndrome. IQR, interquartile range.

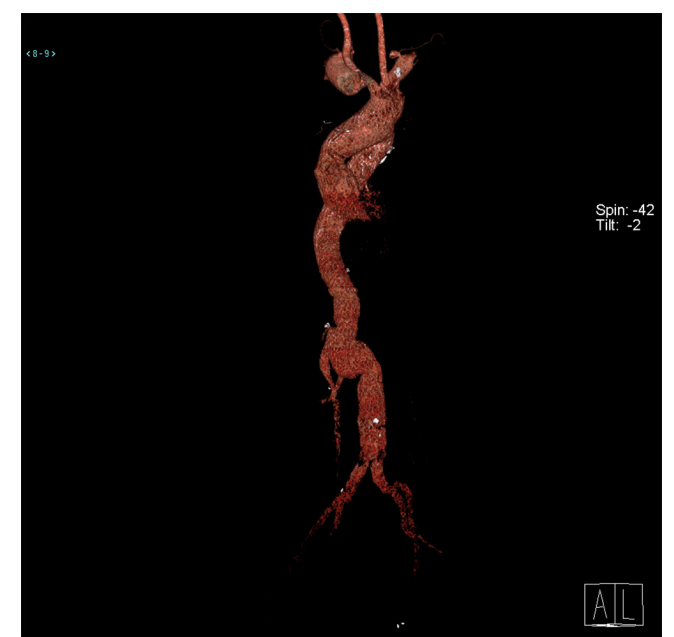

Figure 1 Reconstruction from a 5 -year postoperative CT of a 35-year-old patient with Marfan syndrome and type A aortic dissection, who was submitted to multiple interventions for aortic valve-sparing, ascending-arch-descending-thoracoabdominalabdominal aortic graft replacement. Of note, the native supraaortic trunks and Carrel patch for visceral and renal reimplantation have dilated during follow-up.

\section{Management of type A dissection}

In type A dissection patients with or without Marfan syndrome, open surgery was the preferred treatment method and was performed in $88.4 \%$ and $86.7 \%$ of cases, respectively. Surgical treatment more frequently included a complete arch replacement (Figure 1) in patients with Marfan syndrome compared to those without $(34.1 \%$ vs. $19.6 \% ; \mathrm{P}=0.019)$. Concomitant aortic valve replacement was seen at similar frequency in both groups $(37.5 \%$ vs. $32.2 \%$; $\mathrm{P}=0.483)$, but Marfan patients more commonly received a mechanical valve $(78.6 \%$ vs. $49.9 \%)$ or homograft $(7.1 \%$ vs. $1.9 \%)$ and less commonly a biological valve $(14.3 \%$ vs. $48.2 \% ; \mathrm{P}=0.013)$. Valve-sparing techniques were used in a similar percentage of patients $(24.4 \%$ vs. $19.7 \%$; $\mathrm{P}=0.462)$. Medical management was adopted in a minority of cases, while endovascular or hybrid procedures were very rarely performed (Table 4).

\section{Management of type B dissections}

The approach to type B dissections showed significant 


\begin{tabular}{|c|c|c|c|}
\hline \multicolumn{4}{|l|}{ Type A dissection } \\
\hline Medical management & $15(9.1 \%)$ & 395 (9.9\%) & 0.753 \\
\hline Surgical management & $145(88.4 \%)$ & $3,462(86.7 \%)$ & 0.531 \\
\hline Hybrid management & $2(1.2 \%)$ & $58(1.5 \%)$ & 1.000 \\
\hline \multicolumn{4}{|l|}{ Type B dissection } \\
\hline Medical management & $47(50.0 \%)$ & $1,360(62.6 \%)$ & 0.014 \\
\hline Surgical management & 27 (28.7\%) & $210(9.7 \%)$ & $<0.001$ \\
\hline
\end{tabular}

differences between patients with or without Marfan syndrome. Patients with Marfan syndrome were more frequently treated with open surgery than those without $(28.7 \%$ vs. $9.7 \% ; \mathrm{P}<0.001)$ and less frequently with medical management $(50.0 \%$ vs. $62.6 \% ; \mathrm{P}=0.014)$. Endovascular and hybrid management were adopted at similar frequencies (Table 4).

\section{In-hospital outcomes}

The overall in-hospital mortality rate was $10.9 \%$ in patients with Marfan syndrome compared to $16.9 \%$ in patients without Marfan syndrome $(\mathrm{P}=0.010)$. Table 5 shows the rates of mortality and neurological complications (stroke and/or spinal cord ischemia) by type of dissection and treatment. For patients with type A dissection who underwent open surgical treatment, there was no significant difference in mortality between patients with or without Marfan syndrome (13.1\% vs. $16.6 \% ; \mathrm{P}=0.265)$. For those who received medical treatment, mortality was very high in both groups, but lower in patients with Marfan syndrome than in patients without Marfan syndrome, however, this did not reach statistical significance $33.3 \%$ vs. $55.4 \%$; $\mathrm{P}=0.091)$. In the cohort of patients with Marfan syndrome who underwent open surgical treatment for acute type B dissections $(n=27)$, there were no mortalities, while the mortality rate in those without Marfan syndrome $(n=210)$ was $17.6 \%(\mathrm{P}=0.011)$. The mortality rates of type $\mathrm{B}$ dissection patients treated with medical, endovascular, and hybrid treatment were also lower in patients with Marfan syndrome than in those without, however, this difference was not statistically significant (see Table 5). Neurological complications occurred only in the surgically treated patients with Marfan syndrome (8.2\% for type A dissection and $7.7 \%$ for type $\mathrm{B}$ dissection). However, there were no significant differences in the incidence of neurological complications in the cohort without Marfan syndrome.

\section{Five-year outcomes}

Figure 2 shows 5 -year survival rates of patients with and without Marfan syndrome. There was an initial survival advantage for patients with Marfan syndrome, lost after a follow-up duration of about 2 years. At 5 years followup, there was no significant difference in survival between the two groups $(80.1 \%$ vs. $79.8 \% ; \mathrm{P}=0.712)$. Figure 3 shows reintervention rates of patients with and without Marfan syndrome. Patients with Marfan syndrome more frequently needed to undergo reinterventions, starting within the first year of follow-up. At 5 years follow-up, the difference between both groups in estimated freedom from reintervention rate was significant $(44.7 \%$ vs. $81.5 \%$; $\mathrm{P}<0.001)$. The 5 -year freedom from reintervention rate was $54.4 \%$ vs. $88.0 \%(\mathrm{P}<0.001)$ for patients initially treated with open surgery, $40.0 \%$ vs. $71.9 \%(\mathrm{P}=0.071)$ for those with initial endovascular treatment and $32.0 \%$ vs. $71.5 \%$ $(\mathrm{P}<0.001)$ for patients with initial medical management.

\section{Perspectives}

The current updated IRAD analysis confirms a number of the typical characteristics of Marfan syndrome. First of 


\begin{tabular}{|c|c|c|c|}
\hline Variable & Marfan syndrome & No Marfan syndrome & $P$ value \\
\hline \multicolumn{4}{|l|}{ Type A } \\
\hline \multicolumn{4}{|l|}{ Death } \\
\hline Medical & $5(33.3 \%)$ & $219(55.4 \%)$ & 0.091 \\
\hline Surgical & $19(13.1 \%)$ & $575(16.6 \%)$ & 0.265 \\
\hline Endovascular & $1(50.0 \%)$ & 28 (38.9\%) & 1.000 \\
\hline Hybrid & $0(0.0 \%)$ & $8(13.8 \%)$ & 1.000 \\
\hline \multicolumn{4}{|c|}{ Neurological complications } \\
\hline Medical & $0(0.0 \%)$ & $3(7.7 \%)$ & 1.000 \\
\hline Surgical & $10(8.2 \%)$ & $311(10.5 \%)$ & 0.420 \\
\hline Endovascular & $0(0.0 \%)$ & $12(20 \%)$ & 1.000 \\
\hline Hybrid & $0(0.0 \%)$ & $7(13.2 \%)$ & 1.000 \\
\hline \multicolumn{4}{|l|}{ Type B } \\
\hline \multicolumn{4}{|l|}{ Death } \\
\hline Medical & $2(4.3 \%)$ & $106(7.8 \%)$ & 0.576 \\
\hline Surgical & $0(0.0 \%)$ & $37(17.6 \%)$ & 0.011 \\
\hline Endovascular & $1(5.6 \%)$ & $61(11.1 \%)$ & 0.708 \\
\hline Hybrid & $0(0.0 \%)$ & $7(14.3 \%)$ & 1.000 \\
\hline \multicolumn{4}{|c|}{ Neurological complications } \\
\hline Medical & $0(0.0 \%)$ & $0(0.0 \%)$ & N/A \\
\hline Surgical & $2(7.7 \%)$ & $24(13.3 \%)$ & 0.542 \\
\hline Endovascular & $0(0.0 \%)$ & $51(10.3 \%)$ & 0.380 \\
\hline Hybrid & $0(0.0 \%)$ & $4(10.0 \%)$ & 1.000 \\
\hline
\end{tabular}

all, it shows that dissection in Marfan syndrome occurs in patients at a younger age ( $37 v s$. 62 years for type A; $40 v s$. 64 years for type B). This has also been shown in a previous IRAD study, focusing on patients $<40$ years enrolled in the registry, which found that these patients were more likely to have Marfan syndrome (as well as bicuspid aortic valve and previous aortic surgery) than patients $>40$ years (7). Because of this younger age, Marfan syndrome patients are less frequently affected by atherosclerosis and hypertension at the time of dissection.

Since Marfan syndrome is an autosomal dominant genetic disorder, it is to be expected that it affects men and women in equal proportions. However, we found that only about $35 \%$ of patients with aortic dissection were female, which was not different for patients with Marfan syndrome compared to those without, despite the higher incidence of pregnancy-associated dissection in Marfan syndrome. Recently published data from a Danish national registry of Marfan syndrome patients confirm that male patients have a $75 \%$ higher risk of an aortic event than female patients at any given age (9). Aortic dilatation does not appear to be more prevalent in male than in female adolescents with Marfan syndrome (10). It is not clear why aortic dissection in Marfan syndrome occurs more frequently in men, but there are several hypotheses, including a still unknown protective effect of the $\mathrm{X}$ chromosome in women (11). This is suggested by the fact that women with a deficiency of the $\mathrm{X}$ chromosome (Turner syndrome) have a significantly increased risk of aortic disease, including dissection (12).

We noted significantly larger diameters of the aortic 


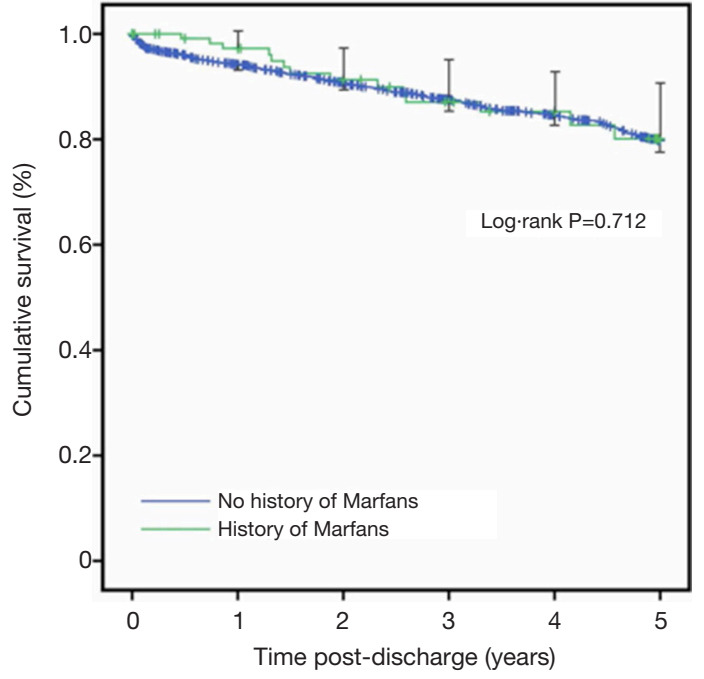

No. at risk

$\begin{array}{lllllll}\text { No. history of Marfans } & 2,205 & 2,104 & 1,590 & 1,242 & 950 & 563 \\ \text { History of Marfans } & 119 & 102 & 76 & 59 & 46 & 20\end{array}$

Figure 2 Kaplan-Meier curves showing the estimated survival rates with $95 \%$ confidence intervals of aortic dissection patients with and without Marfan syndrome up to a follow-up duration of 5 years.

annulus and root in the Marfan syndrome cohort, but not larger diameters more distally. This supports that aortic dilatation in Marfan syndrome involves the annulus and root predominantly. The increased diameter of the aortic annulus is associated with a higher incidence of aortic valve insufficiency. We did not note a difference between Marfan and no Marfan syndrome in the frequency of concomitant aortic valve replacement during surgery for aortic dissection, but this is probably due to the fact that almost a third of patients with Marfan syndrome had already undergone an aortic valve replacement previously. Interestingly, while the aortopathy in Marfan syndrome manifests itself mainly in the proximal aorta, we found no difference in the distribution of type A and B dissection between the cohorts with and without Marfan syndrome.

Another interesting observation is that the incidence of aortic rupture appears to be lower in Marfan syndrome, as suggested by the lower incidence of pericardial effusion and periaortic hematoma on preoperative imaging. One can speculate about the cause. It might be due to the interval time from onset of symptoms to diagnosis, which was significantly shorter in Marfan syndrome (median $4.1 \mathrm{vs}$. 5.0 hours, $\mathrm{P}=0.014$ ), however, the difference in timing of intervention (for type A dissection) did not reach statistical

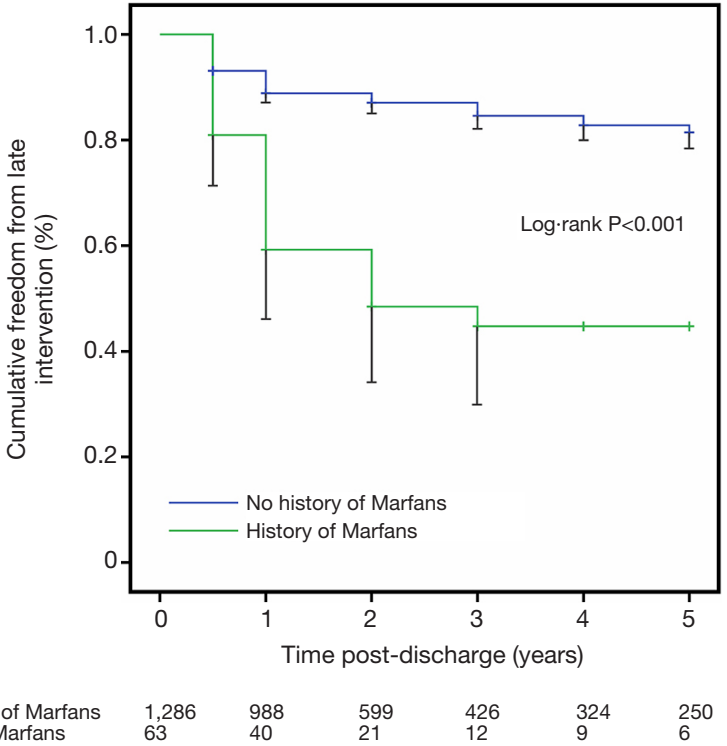

Figure 3 Kaplan-Meier curves showing the estimated freedom from reintervention rates with $95 \%$ confidence intervals of aortic dissection patients with and without Marfan syndrome up to a follow-up duration of 5 years.

significance (8.2 vs. 9.5 hours, $\mathrm{P}=0.117)$.

The in-hospital mortality after surgical treatment of type A dissections was not significantly different for patients with or without Marfan syndrome. A potential reason might be the more extensive surgery that Marfan patients require, both proximally at the level of the aortic root, and distally, at the level of the arch. Such longer operations are associated with longer cross clamp times, which is a known predictor for postoperative mortality (13). Results from other studies suggest that initial hemiarch replacement, eventually followed by elective secondary surgery in case of distal adverse aortic events, is an effective treatment approach $(14,15)$.

In contrast, the in-hospital survival of Marfan syndrome patients with type $\mathrm{B}$ dissection appears to be better than that of patients without Marfan syndrome. The cohort that was treated with open surgery seemed to do especially well, with a $0 \%$ mortality rate $(n=27)$, despite the fact that some of the patients may have been enrolled up to twenty years ago. The experience with endovascular treatment of type B dissection in Marfan syndrome is limited, both within IRAD and other published literature, but suggests relatively high complication rates (16). Currently, many experts believe that only after previous graft replacement of parts 
of the aorta, creating safe landing zones, is there a potential role for endovascular therapy in Marfan syndrome. In case medical management fails, open surgery should thus remain first-line therapy for Marfan syndrome patients with type B dissection, even in the endovascular era, unless the risk of open surgery is considered prohibitive or for life-saving complications.

Lastly, follow-up data show that in a patient cohort with a mean age of 38 years, the estimated 5 -year survival rate is $80 \%$ and over half of the patients underwent reinterventions within 5 years. Late (recurrent) dissection, usually in the downstream aorta, has been recognized before as a risk factor for late mortality (3); a recent IRAD study in 204 patients with recurrent aortic dissection found Marfan syndrome to be a strong independent predictor of recurrent aortic dissection (hazard ratio $8.6,95 \%$ confidence interval, $5.8-12.8 ; \mathrm{P}<0.001)(5)$. It is hypothesized that the risk of distal aortic dissection is increased by altered hemodynamics, due primarily to reduced compliance of the aortic root after graft replacement. Progressive aneurysmal dilatation due to the inherent weakness of the aortic wall may be another reason for either proximal or distal reinterventions. Since the reasons for reintervention were not included in the analysis, these hypotheses could not be confirmed or refuted in the current study.

\section{Conclusions}

Observations from IRAD confirm the classic clinical features of aortic dissection in Marfan syndrome. The majority of patients with type A dissection were treated with open surgery, which was associated with a $13.1 \%$ in-hospital mortality rate. Half of the patients with type B aortic dissection could initially be treated medically, while $28.7 \%$ underwent open surgery. The results of open surgical treatment of type B aortic dissections were excellent and in case medical therapy fails, should be preferred above endovascular treatment. Five-year follow-up shows reduced survival and high reintervention rates in patients with Marfan syndrome, highlighting the need for a careful follow-up protocol after the patient survives the acute phase of aortic dissection.

\section{Acknowledgements}

IRAD is supported by grants from Gore Medical Inc. (Flagstaff, Arizona), Medtronic, Inc. (Minneapolis, Minnesota), Terumo Medical (Tokyo, Japan), The Hewlett
Foundation, Ann and Robert Aikens, the University of Michigan Health System, the Varbedian Fund for Aortic Research, and the Mardigian Foundation.

\section{Footnote}

Conflicts of Interest: The authors have no conflicts of interest to declare.

\section{References}

1. Loeys BL, Dietz HC, Braverman AC, et al. The revised Ghent nosology for the Marfan syndrome. J Med Genet 2010;47:476-85.

2. Silverman DI, Burton KJ, Gray J, et al. Life expectancy in the Marfan syndrome. Am J Cardiol 1995;75:157-60.

3. Gott VL, Greene PS, Alejo DE, et al. Replacement of the aortic root in patients with Marfan's syndrome. N Engl J Med 1999;340:1307-13.

4. Hagan PG, Nienaber CA, Isselbacher EM, et al. The International Registry of Acute Aortic Dissection (IRAD): new insights into an old disease. JAMA 2000;283:897-903.

5. Isselbacher EM, Bonaca MP, Di Eusanio M, et al. Recurrent Aortic Dissection: Observations From the International Registry of Aortic Dissection. Circulation 2016;134:1013-24.

6. Siddiqi HK, Luminais SN, Montgomery D, et al. Chronobiology of Acute Aortic Dissection in the Marfan Syndrome (from the National Registry of Genetically Triggered Thoracic Aortic Aneurysms and Cardiovascular Conditions and the International Registry of Acute Aortic Dissection). Am J Cardiol 2017;119:785-9.

7. Januzzi JL, Isselbacher EM, Fattori R, et al. Characterizing the young patient with aortic dissection: results from the International Registry of Aortic Dissection (IRAD). J Am Coll Cardiol 2004;43:665-9.

8. Januzzi JL, Marayati F, Mehta RH, et al. Comparison of aortic dissection in patients with and without Marfan's syndrome (results from the International Registry of Aortic Dissection). Am J Cardiol 2004;94:400-2.

9. Groth KA, Stochholm K, Hove H, et al. Aortic events in a nationwide Marfan syndrome cohort. Clin Res Cardiol 2017;106:105-12.

10. Mueller GC, Stark V, Steiner K, et al. Impact of age and gender on cardiac pathology in children and adolescents with Marfan syndrome. Pediatr Cardiol 2013;34:991-8.

11. Holmes KW, Maslen CL, Kindem M, et al. GenTAC registry report: gender differences among individuals 
with genetically triggered thoracic aortic aneurysm and dissection. Am J Med Genet A 2013;161A:779-86.

12. Carlson $M$, Silberbach $M$. Dissection of the aorta in Turner syndrome: two cases and review of 85 cases in the literature. BMJ Case Rep 2009;2009:bcr0620091998.

13. Doenst T, Borger MA, Weisel RD, et al. Relation between aortic cross-clamp time and mortality--not as straightforward as expected. Eur J Cardiothorac Surg 2008;33:660-5.

14. Rylski B, Bavaria JE, Beyersdorf F, et al. Type A

Cite this article as: de Beaufort HW, Trimarchi S, Korach A, Di Eusanio M, Gilon D, Montgomery DG, Evangelista A, Braverman AC, Chen EP, Isselbacher EM, Gleason TG, De Vincentiis C, Sundt TM, Patel HJ, Eagle KA. Aortic dissection in patients with Marfan syndrome based on the IRAD data. Ann Cardiothorac Surg 2017;6(6):633-641. doi: 10.21037/ acs.2017.10.03 aortic dissection in Marfan syndrome: extent of initial surgery determines long-term outcome. Circulation 2014;129:1381-6.

15. Schoenhoff FS, Kadner A, Czerny M, et al. Should aortic arch replacement be performed during initial surgery for aortic root aneurysm in patients with Marfan syndrome? Eur J Cardiothorac Surg 2013;44:346-51; discussion 351.

16. Pacini D, Parolari A, Berretta P, et al. Endovascular treatment for type B dissection in Marfan syndrome: is it worthwhile? Ann Thorac Surg 2013;95:737-49. 